\title{
CAPÍTULO 01: ALIMENTOS FUNCIONAIS PROBIÓTICOS, UM NOVO ESTILO DE VIDA
}

\section{CHAPTER 01: PROBIOTIC FUNCTIONAL FOOD, A NEW LIFESTYLE}

\author{
Williames Fabio de Souza Bezerra Filho"; Larissa Nunes Torres²; Maria Clara Leal Bezerra Barros ${ }^{3}$; Sonara \\ de França Sousa ${ }^{4}$
}

\begin{abstract}
Resumo
O presente trabalho teve como objetivo traçar o perfil dos consumidores de probióticos por meio de um questionário online composto por 21 perguntas relacionadas ao perfil socioeconômico, de saúde e de consumo, compartilhado via link. De acordo com os resultados obtidos, a maioria são mulheres, autodeclaradas brancas, residentes na região Nordeste, com idade entre 20 a 35 anos, renda mensal de até dois salários mínimos, praticam atividade física com pouca frequência e apresentam algum tipo de doença crônica, com destaque para a hipertensão, obesidade e diabetes. 42,20\% afirmaram saber o significado do termo "alimento funcional", 45,10\% afirmaram saber as características de um alimento probiótico e 58,40\% afirmaram estar preocupados com a saúde ao adquiri-los, sendo os probióticos mais consumidos os queijos, iogurtes, leite fermentado e coalhada. A frequência de consumo foi de 3 a 4 vezes por semana e 36,60\% afirmaram apresentar melhorias no funcionamento do intestino. Sobre a comercialização, $58,26 \%$ da aquisição dos alimentos probióticos é em supermercados, $69,10 \%$ estão dispostos a pagar mais por um alimento saudável e 87,30\% afirmaram que os fabricantes deveriam informar sobre os benefícios e possíveis malefícios do produto nos rótulos, uma vez que a embalagem é um fator decisivo na escolha do produto.
\end{abstract}

Palavras-Chaves: consumidor, funcionamento do organismo, perfil, produtos lácteos, saúde.

\begin{abstract}
This work aimed to outline the profile of probiotic consumers through an online questionnaire composed of 21 questions related to the socioeconomic, health and consumption profile, shared via link. According to the results obtained, the majority are women, self-declared white, residing in the Northeast, aged between 20 and 35 years, monthly income of up to two minimum wages, practice physical activity infrequently and have some type of chronic disease, with emphasis on hypertension, obesity and diabetes. $42.20 \%$ said they knew the meaning of the term "functional food", $45.10 \%$ said they knew the characteristics of a probiotic food and $58.40 \%$ said they were concerned with health when purchasing them, with the most consumed probiotics being cheeses, yogurts, fermented milk and curds. The frequency of consumption was 3 to 4 times a week and $36.60 \%$ said they had improvements in bowel function. Regarding commercialization, $58.26 \%$ of probiotic food purchases are from supermarkets, $69.10 \%$ are willing to pay more for healthy food and $87.30 \%$ stated that manufacturers should inform about the benefits and possible harms of the product in labels, since the packaging is a decisive factor in the choice of the product.
\end{abstract}

\footnotetext{
${ }^{1}$ Engenharia de Alimentos, Universidade Federal do Agreste de Pernambuco, williamesfabio_2001@ outlook.com

${ }^{2}$ Engenharia de Alimentos, Universidade Federal do Agreste de Pernambuco, larissan.torres159@gmail.com

3 Engenharia de Alimentos, Universidade Federal do Agreste de Pernambuco, mclaraleal.engalimentos@gmail.com

4 Doutora em Engenharia de Processos, Universidade Federal do Agreste de Pernambuco, sonara_franca@yahoo.com.br
} 
Keywords: consumer, body functioning, profile, dairy products, health.

\section{Introdução}

O termo alimento funcional surgiu no Japão por volta de 1980, quando o governo japonês iniciou um programa de redução de custos de seguro saúde e medicamentos, em especial voltada à população que estava se tornando mais velha e procurou incentivar qualquer metodologia que pudesse melhorar a perspectiva da saúde em longo prazo (IKEDA et al, 2010). Esse termo é dado a alimentos que além das suas funções básicas nutricionais, apresentam também benefícios fisiológicos ou que reduzem riscos de doenças crônicas (STRINGHETA et al, 2007).

Segundo a Resolução n ${ }^{\circ}$ 19, de 30 de abril de 1999, sobre a alegação de propriedade funcional dos alimentos, é aquela relativa ao papel metabólico ou fisiológico que o nutriente ou não nutriente tem no crescimento, desenvolvimento, manutenção e outras funções normais do organismo humano (BRASIL, 1999). Entre os compostos funcionais mais investigados pela ciência, temos os probióticos, que segundo a RDC n 241, de 26 de julho de 2018, é definido como microrganismos vivos que, quando administrado em quantidades adequadas, conferem benefícios à saúde do indivíduo (BRASIL, 2018).

Esses microrganismos podem ser encontrados em alimentos, bebidas ou em suplementos alimentares, fazendo parte da composição de iogurtes, sucos e bebidas lácteas fermentadas, contribuindo para um mercado significativo. Nos iogurtes e bebidas lácteas, encontramos com mais frequência as bactérias anaeróbicas, Bifidobacteria e Lactobacilli, sendo o iogurte o mais consumido popularmente para obtenção desse microrganismos (SHAIKH, 2018).

Dentre os benefícios trazidos ao homem em função do consumo de alimentos contendo probióticos, estão a síntese de vitaminas e proteínas pré-digeridas, a inibição de patógenos, a reconstituição da microbiota intestinal após o uso de antibióticos, o aumento da imunidade, a redução da atividade ulcerativa, o controle da colite e o possível efeito hipocolesterolêmico, sendo sua presença muito importante nos alimentos (SAAD, 2006; GOLDIN; GORBACH, 2008).

Poucas pesquisas sobre o comportamento dos consumidores de alimentos probióticos são encontradas na literatura, diante da importância dos fatores relacionados ao conhecimento, aceitação e compra destes produtos, o presente trabalho teve como objetivo traçar o perfil dos consumidores de alimentos probióticos por meio de um questionário online compartilhado via link em redes sociais. 


\section{Material e Métodos}

A metodologia abordada foi através da utilização de um questionário online elaborado na plataforma Google ${ }^{\circledR}$ Forms, compartilhado nas redes sociais via link e atingiu um grupo de 457 pessoas. O questionário continha 20 perguntas de múltipla escolha e 1 aberta, onde foi realizado um teste de triangulação para se obter as respostas mais comentadas pelos participantes.

Para caracterizar os participantes, foram incluídas questões sobre gênero, raça/etnia, lugar do país onde mora, faixa etária e renda familiar, além de informações relacionadas a saúde dos participantes, quanto a de prática de atividades físicas e doenças crônicas. Quanto ao conhecimento e uso de probióticos foram abordadas perguntas tais como: a frequência do consumo de probióticos, a preocupação com o consumo de alimentos mais saudáveis, locais que costumam encontram os produtos, porque os consome, quais os mais consumidos. Além de perguntar se conhecem o que é um produto funcional e um produto probiótico, e ainda se percebe melhora ao utilizar esses alimentos. Os dados obtidos foram dispostos em forma de gráficos de barras utilizando o software Excel.

\section{Resultados e Discussão}

De acordo com o perfil socioeconômico dos participantes (Figuras 1, 2, 3, 4 e 5), são em sua maioria mulheres, autodeclaradas brancas, residentes na região Nordeste, com idade entre 20 a 35 anos e renda mensal de até dois salários mínimos. BAYER (2012) ao fazer um levantamento do perfil de consumidores de alimentos funcionais no município de Ponta Grossa - PR, verificou que dos 200 consumidores participantes, 65\% eram do sexo feminino de faixa etária predominante entre 26 a 35 anos (36,5\%), já CRUZ (2016), avaliando o perfil de 124 consumidores de alimentos funcionais, constatou que 58,9\% eram mulheres, de faixa etária entre 25 a 30 anos (29\%) e apresentavam ensino médio completo ou superior incompleto (68\%).

A predominância das mulheres nesse cenário também foi reportado por LEITE (2011) afirmando que os consumidores do sexo feminino são considerados o principal público-alvo de alimentos funcionais, pois demonstram uma postura reflexiva sobre o impacto do consumo de alimentos na sua saúde. 
Figura 1: Gênero dos participantes que responderam o questionário.

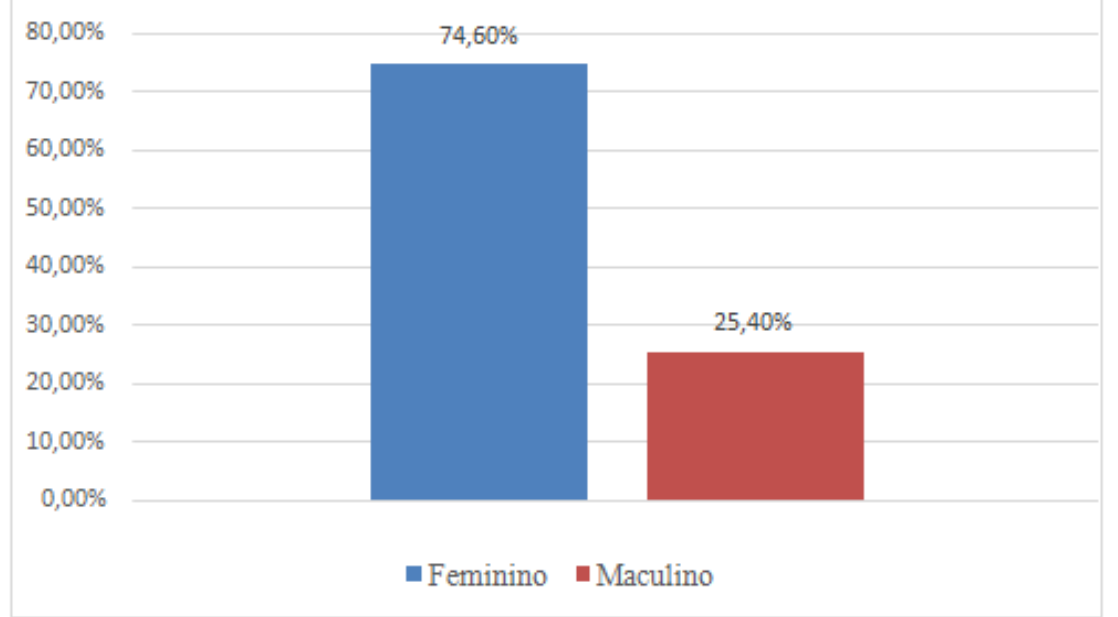

Fonte: Própria (2020)

Figura 2: Raça dos participantes do questionário.

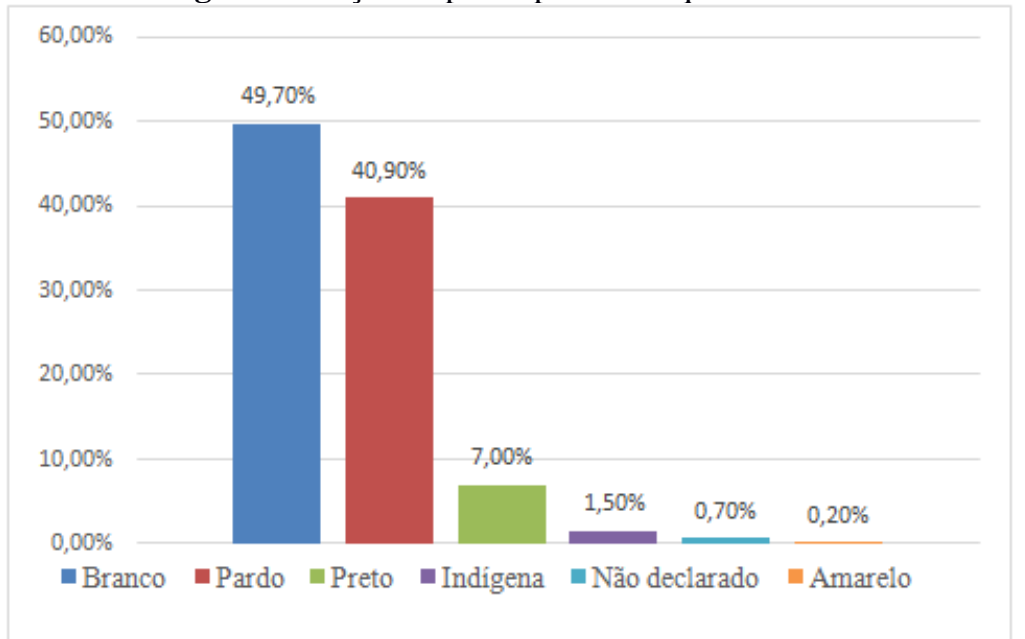

Fonte: Própria (2020)

Figura 3: Região do país onde moram os participantes do questionário.

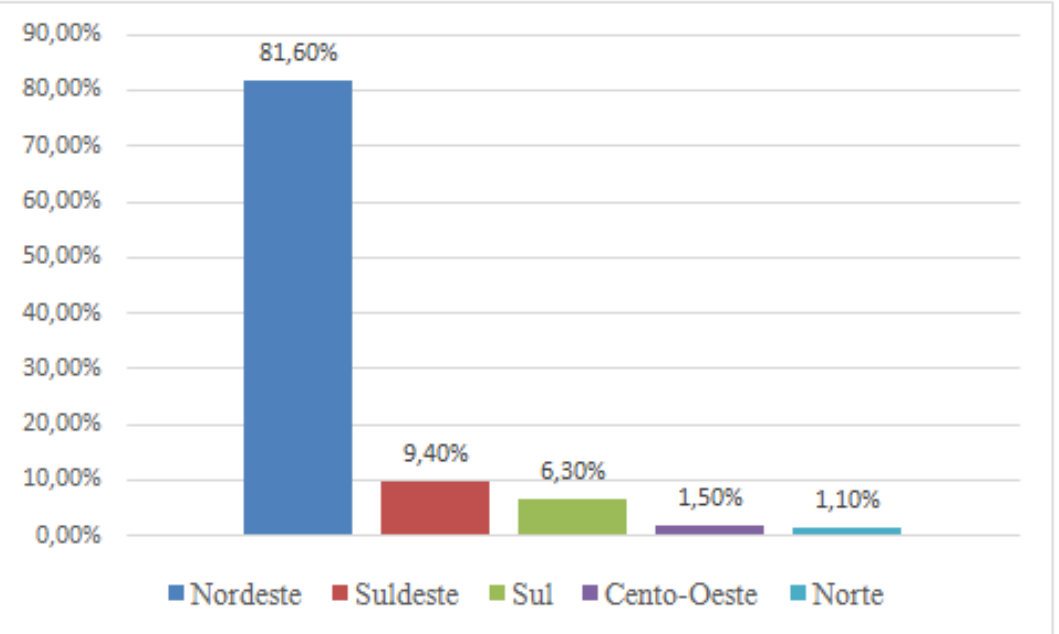

Fonte: Própria (2020)

[4] 
Figura 4: Idade dos participantes do questionário.

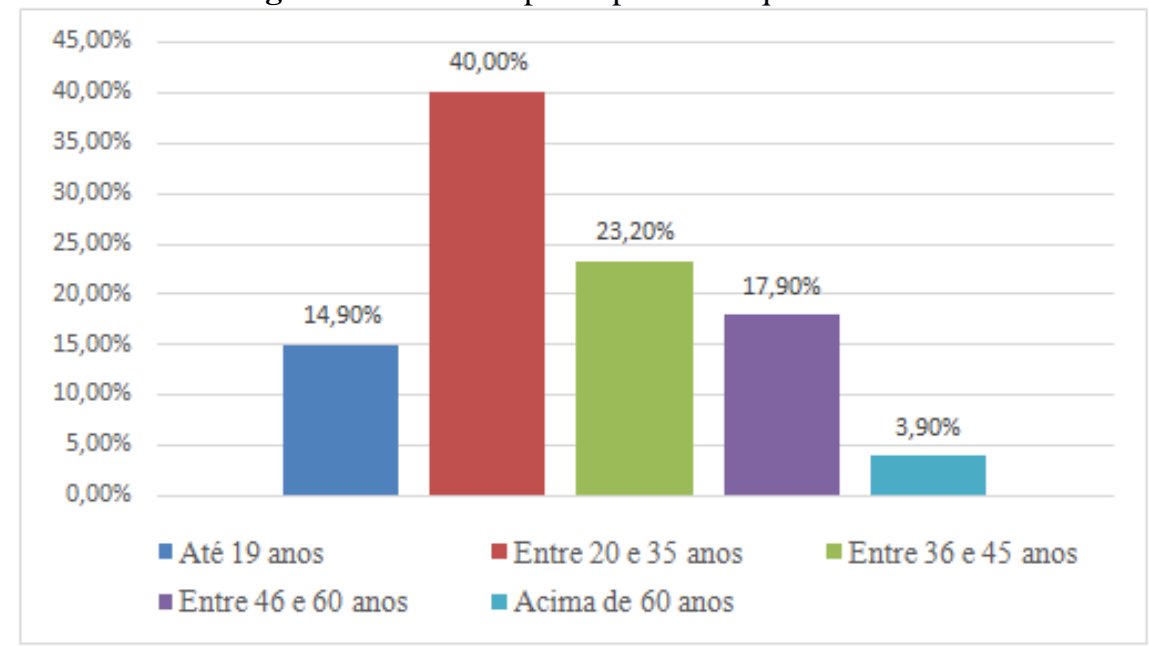

Fonte: Própria (2020)

Figura 5: Renda mensal dos participantes do questionário.

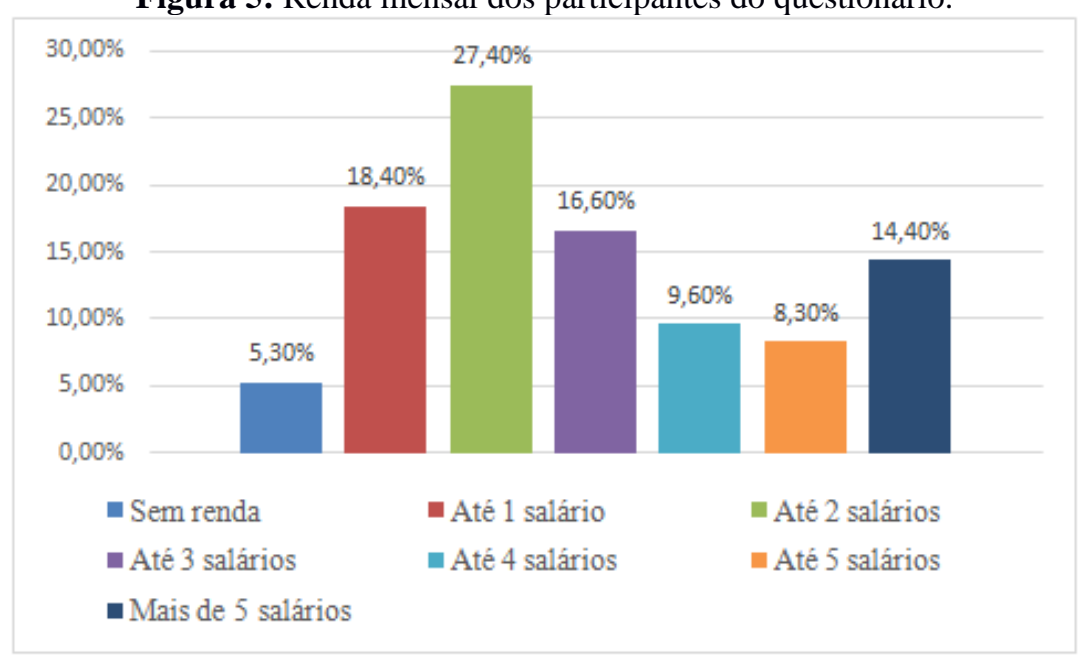

Fonte: Própria (2020)

Quando perguntados sobre a prática de exercícios físicos (Figura 6), 37\% dos entrevistados responderam fazer com pouca frequência. Quando questionados sobre a presença de doenças crônicas (Figura 7), 80,10\% afirmaram ter, com destaque para a hipertensão (28\%) obesidade $(10 \%)$ e diabetes $(7 \%)$, respectivamente (Figura 8). As doenças crônicas não transmissíveis (DCNT), tais como a obesidade, a hipertensão arterial, o diabetes, as neoplasias, as doenças pulmonares obstrutivas crônicas, a osteoporose e as doenças cardiovasculares, podem ter como causa os fatores ambientais, incluindo os hábitos não saudáveis, como a dieta inadequada e o sedentarismo, o que, por sua vez, torna-se uma ameaça à saúde e ao desenvolvimento humano global (Schmidt et al., 2011; Lima, 2012). 
FILHO, et al.

Figura 6: Frequência da prática de exercícios dos participantes.

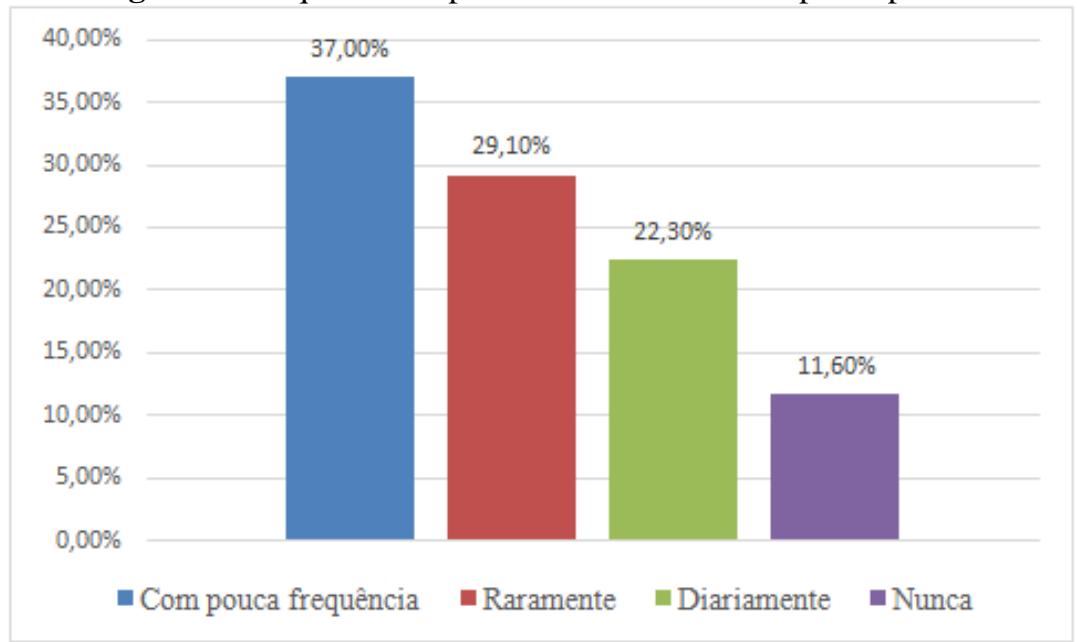

Fonte: Própria (2020)

Figura 7: Disponibilidade de doenças crônicas dos participantes do questionário.

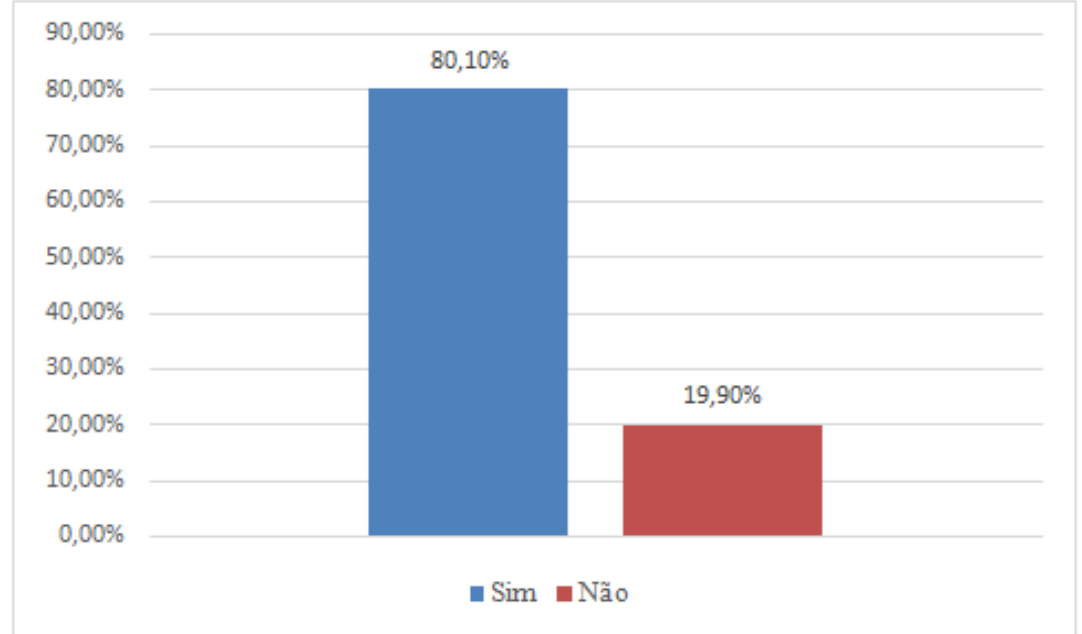

Fonte: Própria (2020)

Figura 8. Doenças crônicas apresentadas

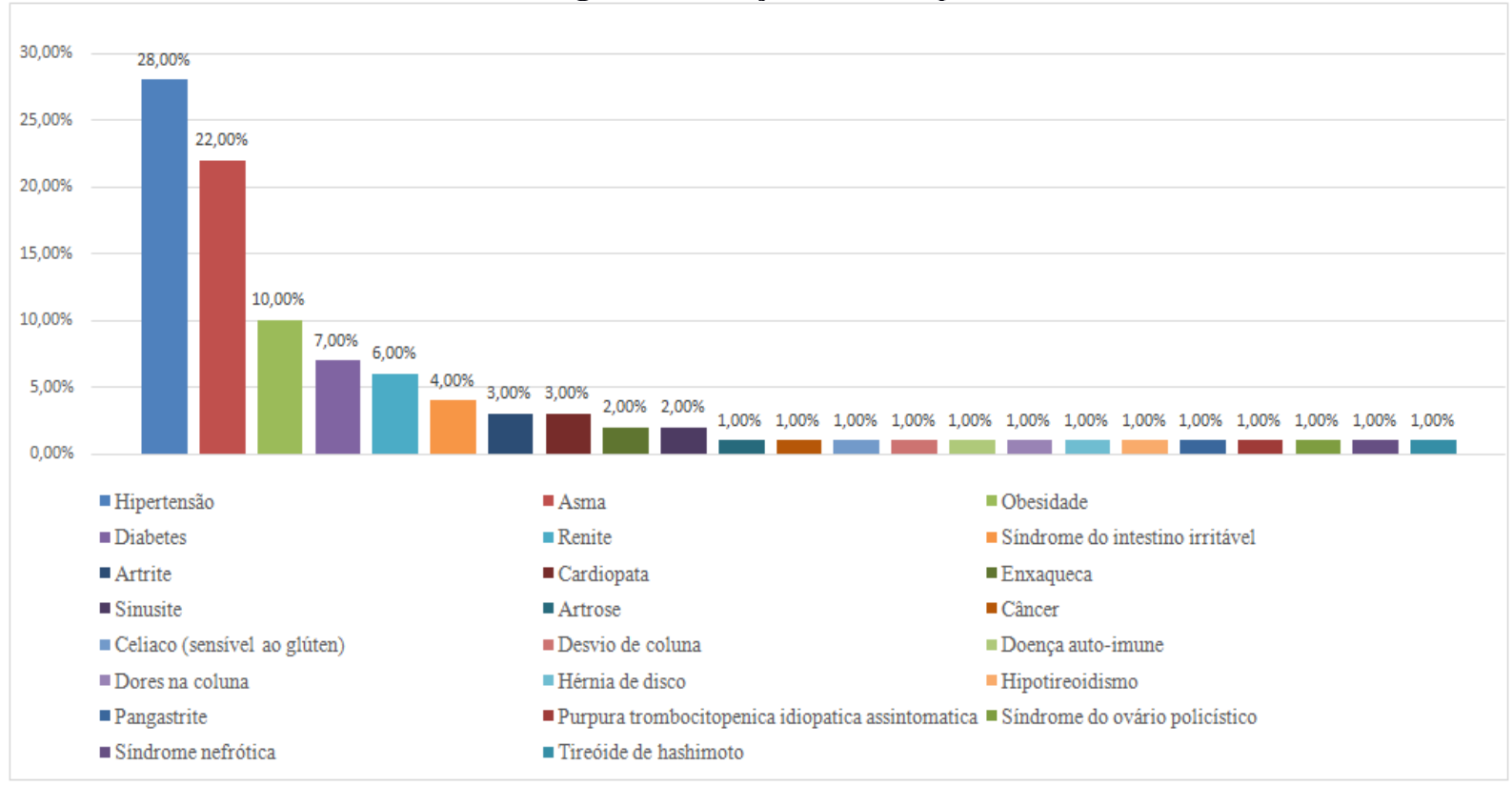

Fonte: Própria (2020)

[6]

CIÊNCIA, TECNOLOGIA E INOVAÇÃO: DO CAMPO À MESA. RECIFE: EDITORA IIDV, 2020 
Quando perguntados sobre o conhecimento do que é um alimento funcional, 42,20\% dos entrevistados responderam saber o que se trata (Figura 9). Os alimentos funcionais caracterizam-se por oferecer vários benefícios à saúde, além do valor nutritivo inerente à sua composição química, podendo desempenhar um papel potencialmente benéfico na redução do risco de doenças crônicas degenerativas (BIBLIOTECA VIRTUAL EM SAÚDE, 2020).

Figura 9: Conhecimento dos participantes sobre o que é um alimento funcional.

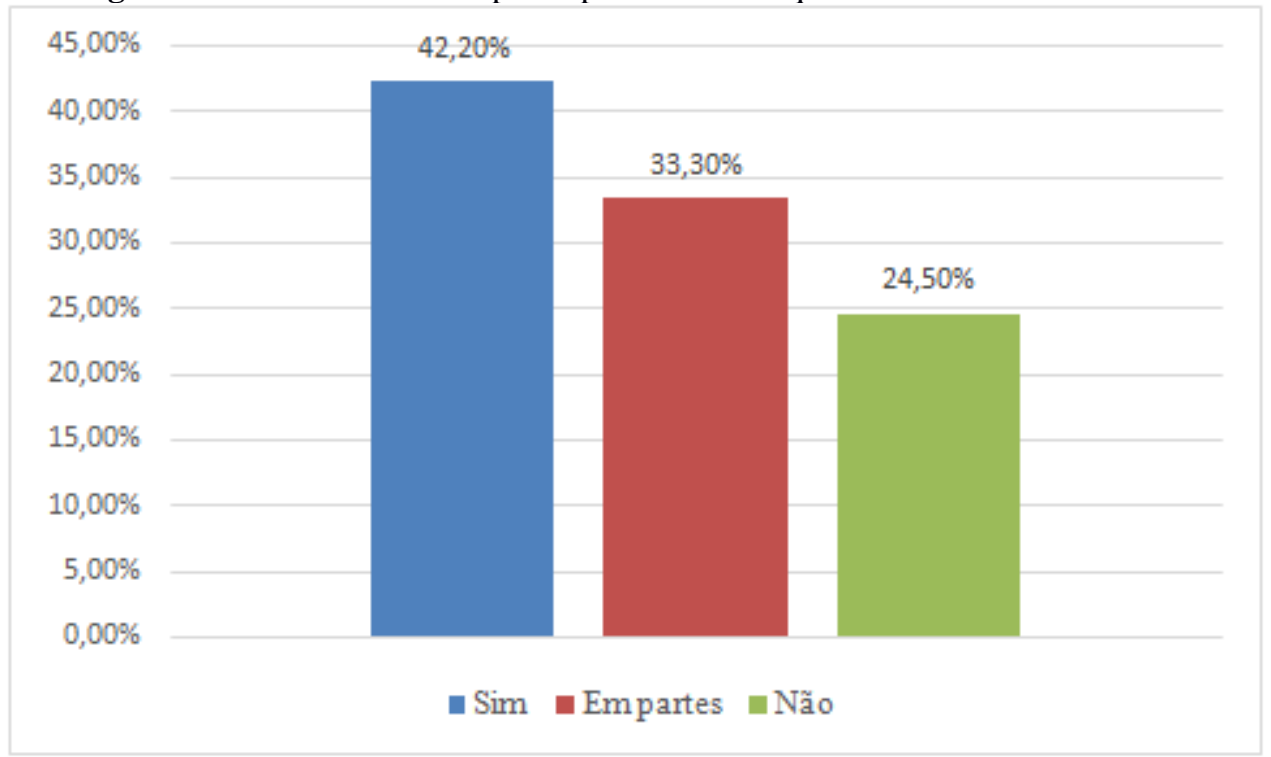

Fonte: Própria (2020)

$\mathrm{Na}$ classe dos alimentos funcionais, encontram-se os probióticos, 45,10\% dos respondentes afirmaram saber as características desse tipo de produto (Figura 10) e 58,40\% afirmaram estar preocupados com a saúde ao adquirir-los (Figura 11).

Quando perguntados sobre o tipo de probiótico mais consumidos (Figura 12), observouse que os produtos lácteos fermentados apresentaram destaque, a saber, os queijos $(35,57 \%)$, iogurtes $(31,88 \%)$, leite fermentado $(13,58 \%)$ e coalhada $(8,21 \%)$. Os derivados lácteos fermentados apresentam propriedades terapêuticas por incluírem em sua composição bactérias como Lactobacillus e Bifidobacterium que possuem características probióticas, e essas apresentam efeitos bioquímicos sobre os nutrientes do leite e efeitos fisiológicos sobre o consumidor (FARIA et al., 2006; GALLINA et al., 2012; LEUCAS, 2012).

Também foi observado que alguns respondentes mencionaram consumir alimentos que não são considerados probióticos, embora também apresente propriedades funcionais, tais como a chia e a linhaça, estes grãos são classificados como prebióticos, ou seja, são alimentos não digeríveis pelo hospedeiro (fibras) e que tem a propriedade de serem fermentados de maneira seletiva no cólon, favorecendo o seu bem-estar (FERREIRA; SILVA, 2010). 
FILHO, et al.

Figura 10: Conhecimento dos participantes sobre o que é um alimento probiótico.

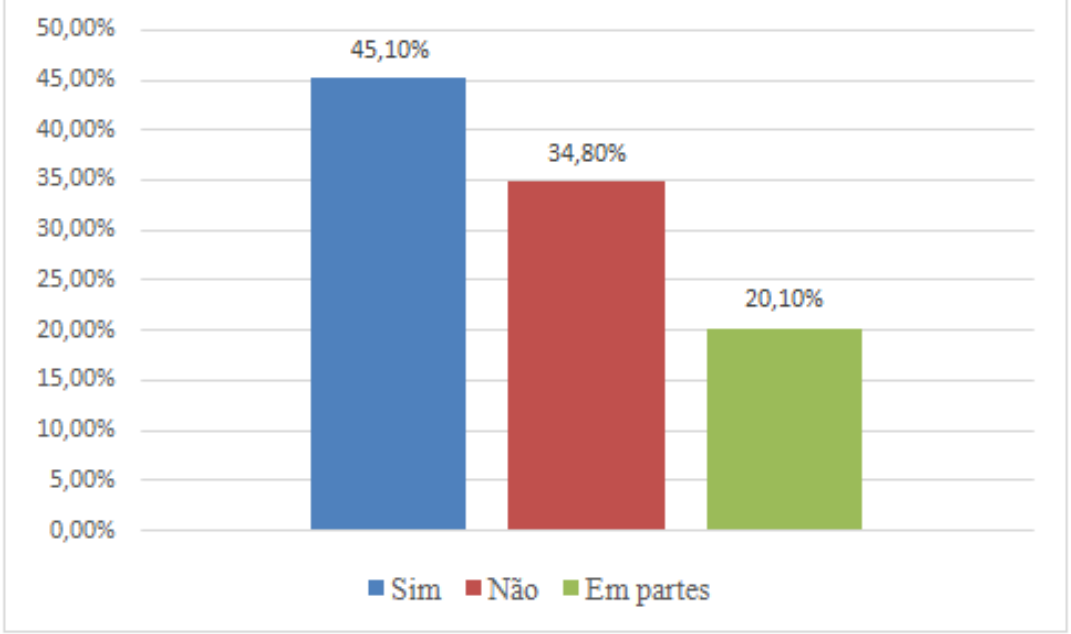

Fonte: Própria (2020)

Figura 11: Preocupação dos participantes em comprar alimentos mais saudáveis.

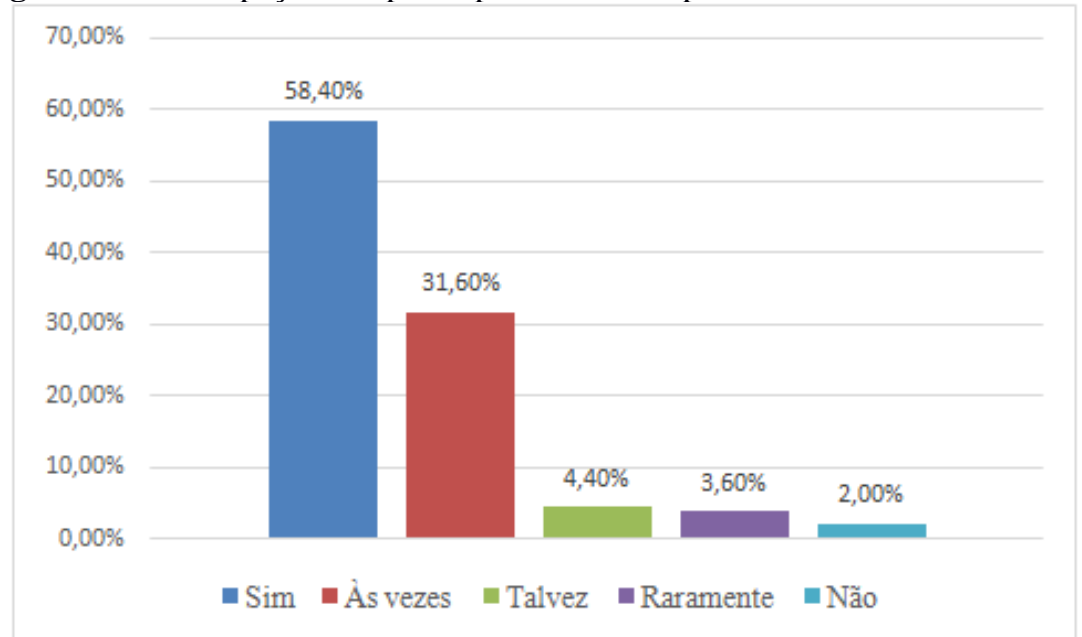

Fonte: Própria (2020)

Figura 12: Quais os produtos mais consumidos?

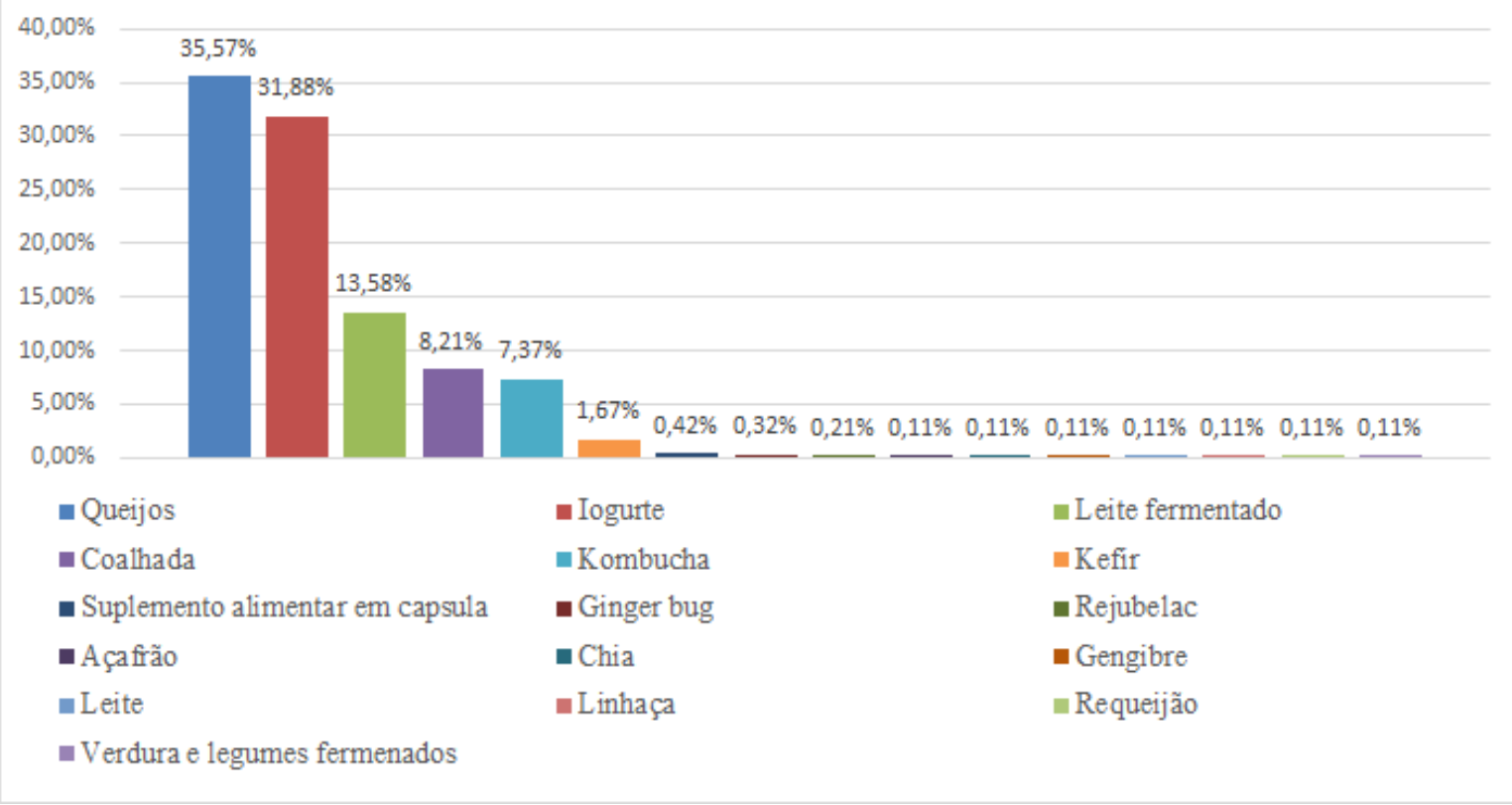

Fonte: Própria (2020)

$[8]$ 
A Figura 13 refere-se ao motivo dos participantes em consumirem os probióticos mencionados na figura anterior. Percebe-se que o "sabor" foi mencionado em 89 das respostas registradas, referindo-se à aceitação dos probióticos por parte deste atributo, seguidos pelas palavras "saúde e saudáveis" e "bom funcionamento do intestino".

A figura 14 refere-se a intenção na hora da compra do alimento probiótico, 60,20\% responderam ser o sabor e $26,50 \%$ responderam por ser um alimento saudável, correlacionado com as respostas da Figura 13.

Figura 13: Principais motivos descritos pelos participantes ao consumirem um alimento probiótico.

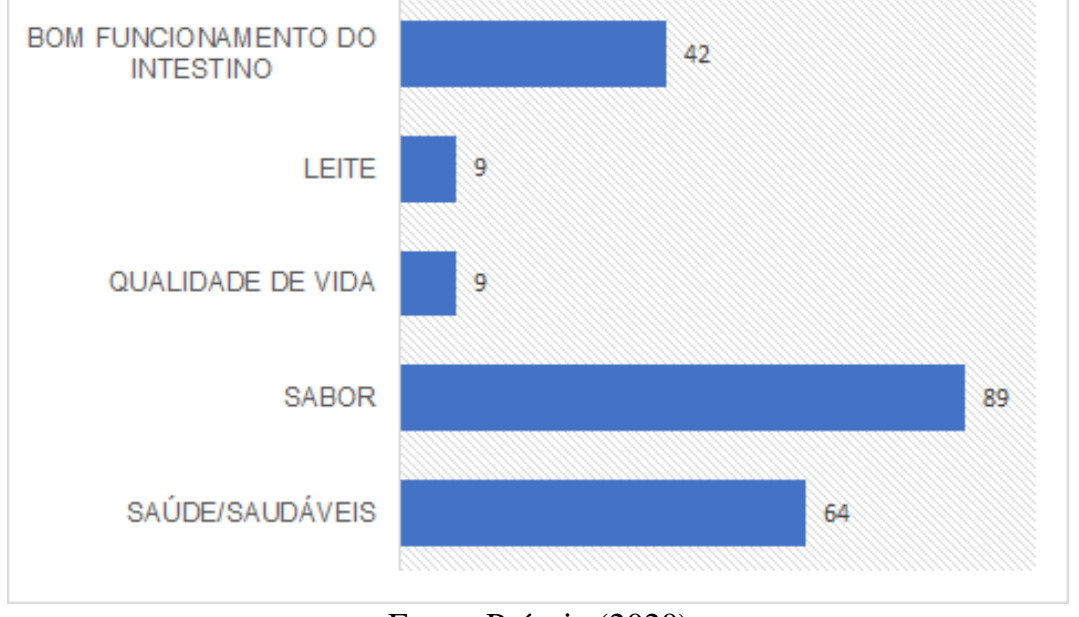

Fonte: Própria (2020)

Figura 14: O que é levando em conta na hora da compra de um alimento probiótico

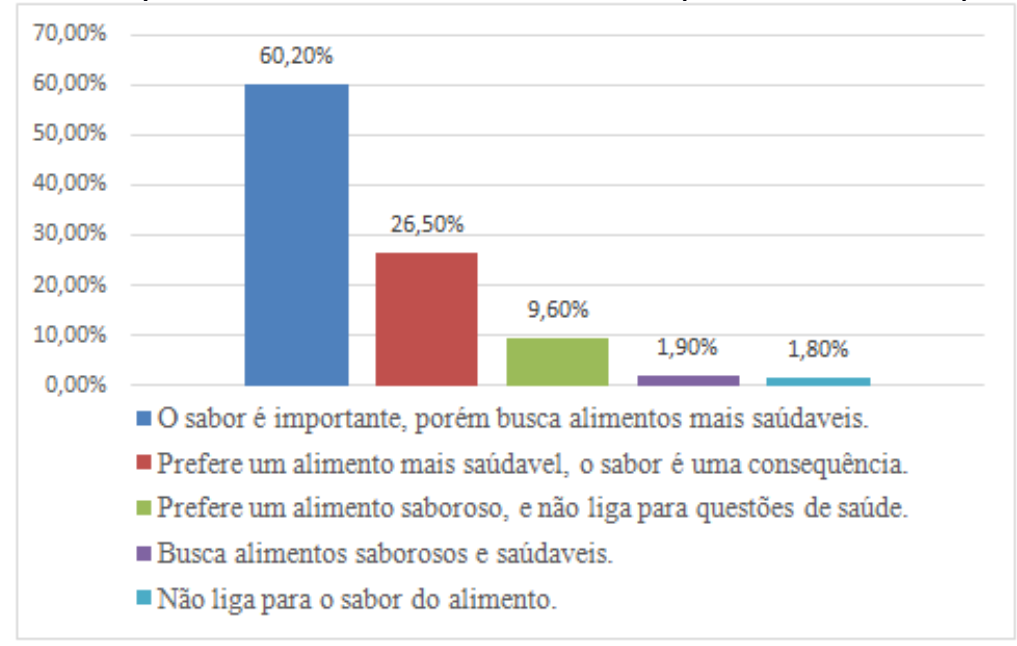

Fonte: Própria (2020)

Quando perguntados sobre a frequência em que consomem probióticos (Figura 15), 37,90\% responderam ser de 3 a 4 vezes por semana. Sobre a ação dos lactobacillus vivos para regularizar o intestino (Figura 16), 44,50\% responderam não fazer uso. Foi perguntado aos participantes que fazem o uso de probióticos para regular o intestino, se eles notaram as melhorias ao consumirem o produto (Figura 17), 36,60\% afirmaram que sim, corroborando com BAYER (2012) ao mencionar que os principais benefícios apontados pelos consumidores de 
FILHO, et al.

alimentos probióticos é a melhora da função intestinal $(34,4 \%)$ e o fortalecimento do sistema imunológico $(29,1 \%)$.

Figura 15: Frequência do consumo de probióticos, pelos participantes do questionário.

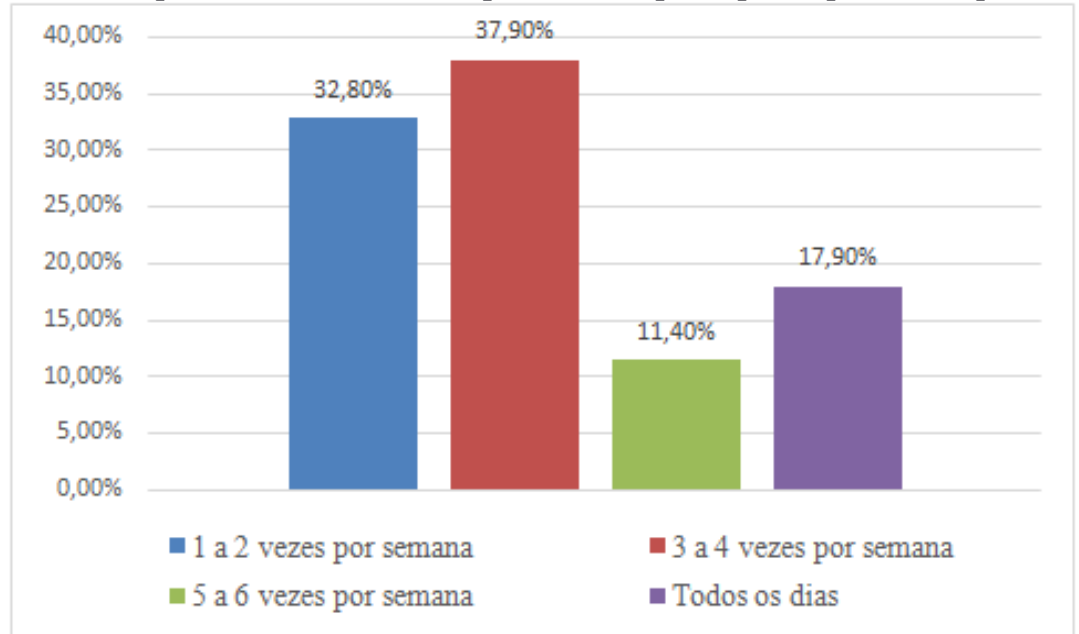

Fonte: Própria (2020)

Figura 16: Uso de lactobacilos vivos para regularizar o intestino, pelos participantes do questionário.

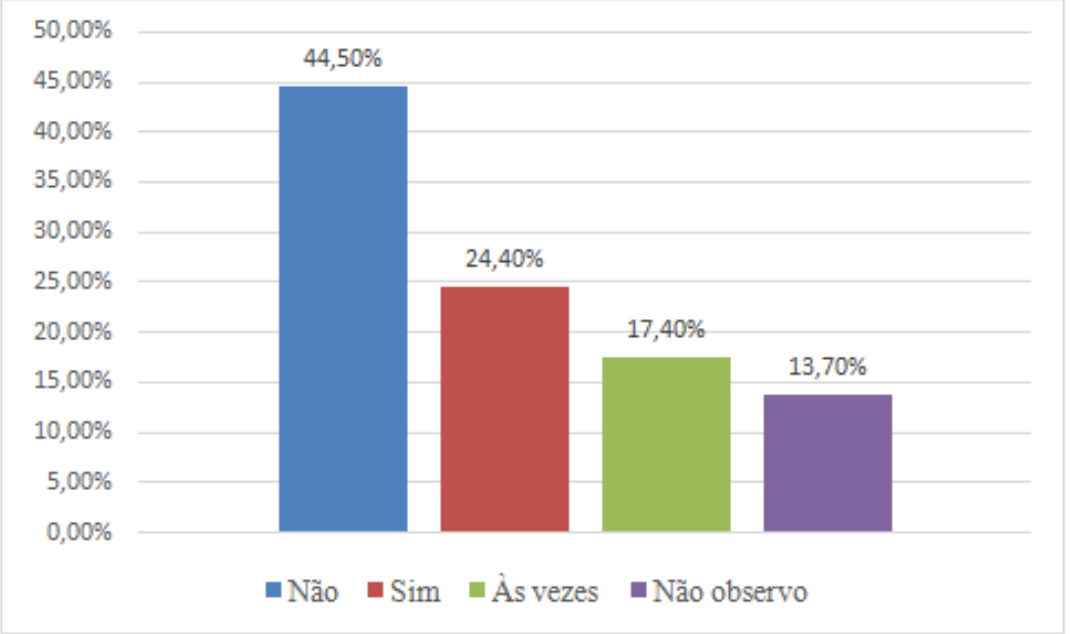

Fonte: Própria (2020)

Figura 17: Participantes que notam melhorias ao utilizar probióticos para regular o intestino.

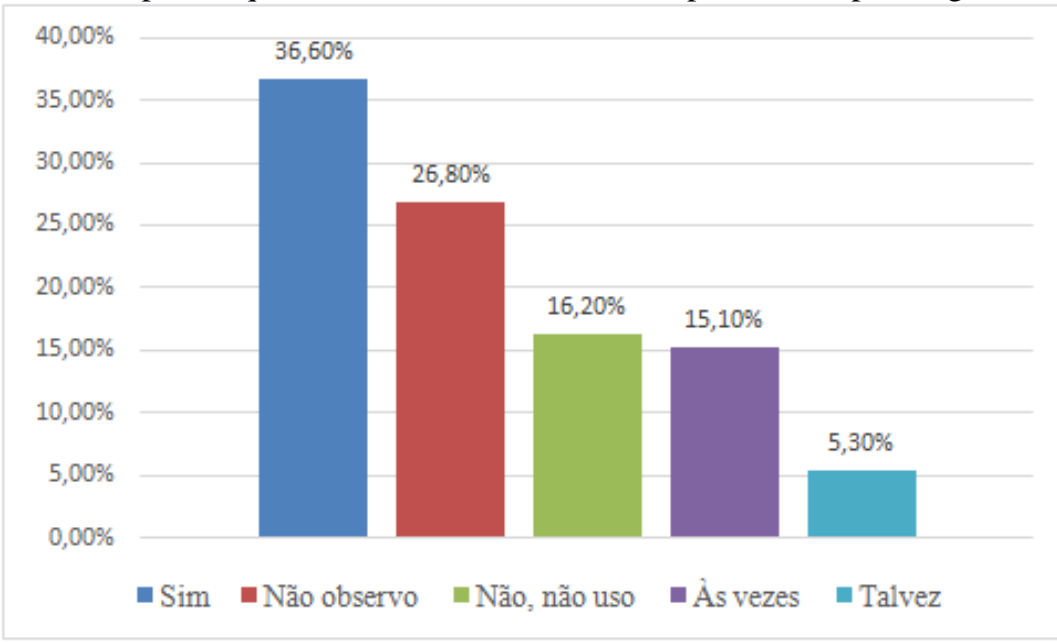

Fonte: Própria (2020) 
Sobre a comercialização dos alimentos probióticos, 58,26\% afirmaram adquiri-los em supermercados (Figura 18); 69,10\% responderam que pagariam a mais por um alimento saudável (Figura 19); e 68,50\% afirmaram faltar incentivo dos mercados em fornecer alimentos mais saudáveis aos consumidores (Figura 20). GALLOTTI (2012) afirma que os consumidores se preocupam com os custos dos produtos funcionais quando eles são para consumo familiar e que estão dispostos a pagar preços mais altos pelos mesmos, pois, ao adquiri-los não estão levando para casa apenas o produto em si, mas sim, levando saúde e qualidade de vida por meio de todos os benefícios inseridos na composição do alimento.

Quando questionados sobre a rotulagem dos probióticos (Figura 21) 87,30\% afirmaram que os fabricantes deveriam informar mais sobre os benefícios e possíveis malefícios do produto corroborando com LEITE (2011) ao mencionar que os consumidores utilizam os rótulos para obter informações referentes aos benefícios e características dos alimentos funcionais mais do que outros veículos como os sites na internet ou propagandas na televisão.

Figura 18: Onde os participantes do questionário costumam encontrar alimentos probióticos.

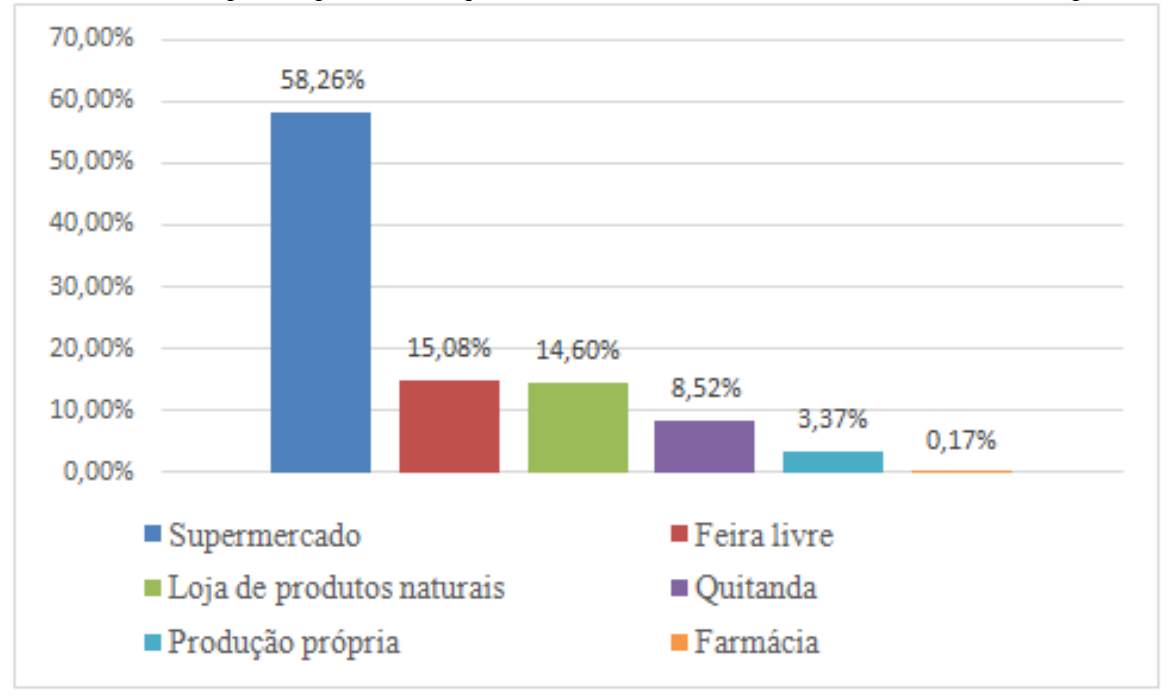

Fonte: Própria (2020) 
Figura 19: Participantes que estariam dispostos a pagar mais por alimentos mais saudáveis.

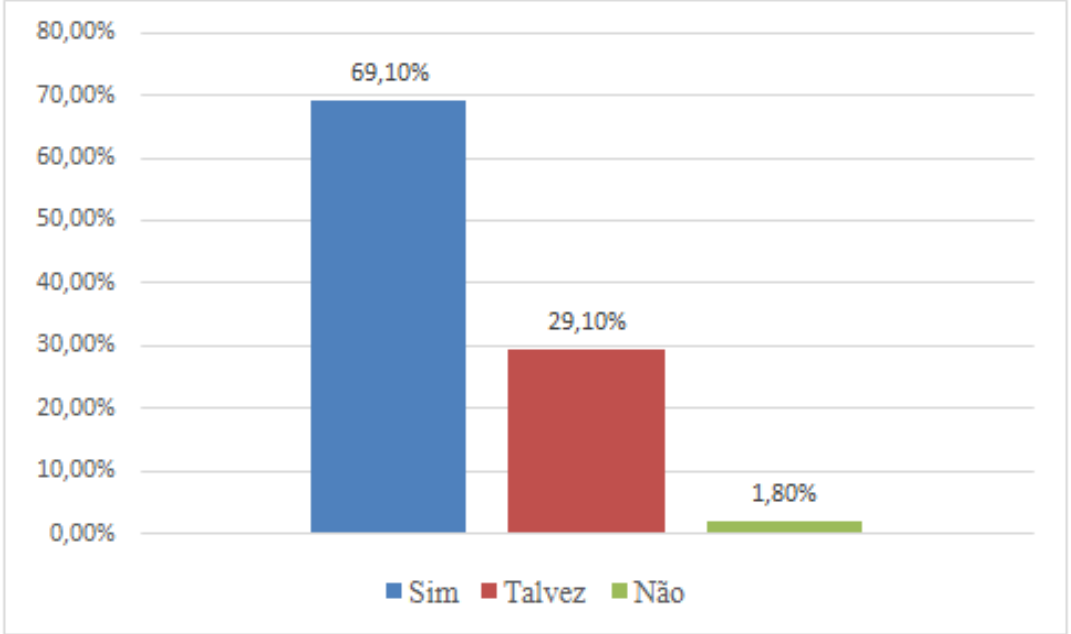

Fonte: Própria (2020)

Figura 20: Falta incentivo dos mercados para comprar alimentos mais saudáveis?

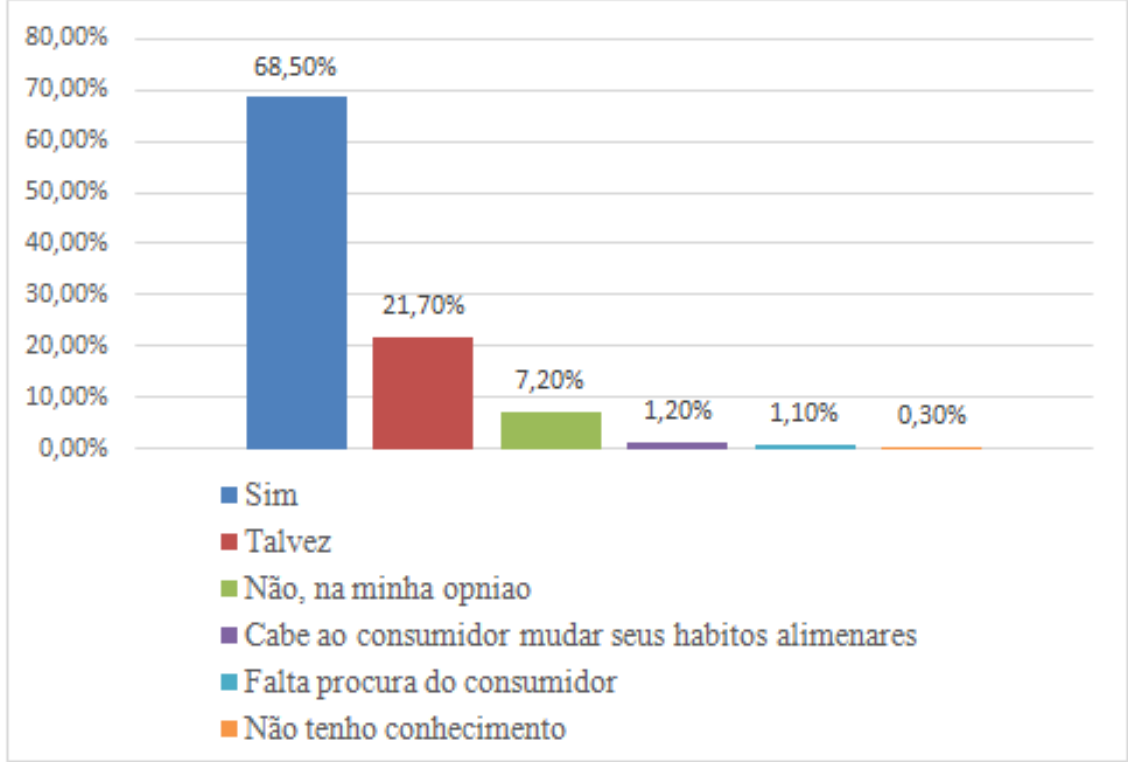

Fonte: Própria (2020)

Figura 21: Importância de ter um rótulo mais claro, de acordo com os participantes do questionário.

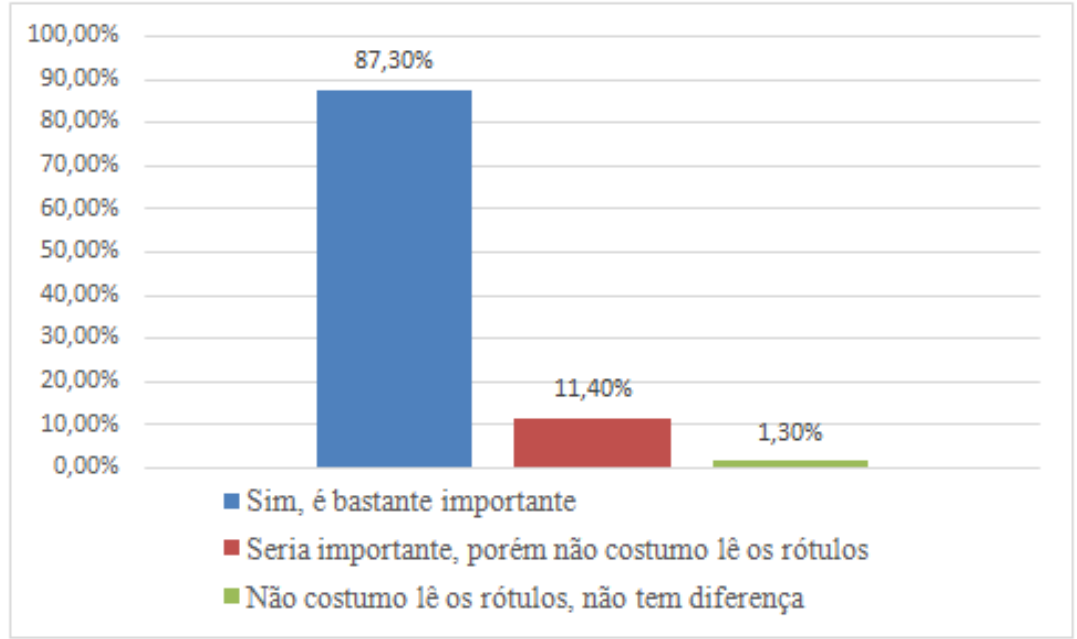

Fonte: Própria (2020) 


\section{Conclusões}

De acordo com o perfil dos participantes, são em sua maioria mulheres, autodeclaradas brancas, residentes na região Nordeste, com idade entre 20 a 35 anos, renda mensal de até dois salários mínimos, praticam atividade física com pouca frequência e apresentam algum tipo de doença crônica, com destaque para a hipertensão, obesidade e diabetes.

Sobre o conhecimento de alimentos funcionais, 42,20\% dos entrevistados afirmaram saber o significado do termo, 45,10\% afirmaram saber as características de um alimento probiótico e 58,40\% afirmaram estar preocupados com a saúde ao adquirir-los, sendo os probióticos mais consumidos os queijos, iogurtes, leite fermentado e coalhada.

O sabor é o principal atributo relacionado à aceitação dos probióticos, seguido pelas características de serem considerados alimentos saudáveis. A frequência de consumo foi de 3 a 4 vezes por semana e $36,60 \%$ afirmaram que apresentam melhorias no funcionamento do intestino.

Sobre a comercialização, 58,26\% da aquisição dos alimentos probióticos é em supermercados, $69,10 \%$ dos participantes estão dispostos a pagar mais por um alimento saudável e 87,30\% afirmaram que os fabricantes deveriam informar mais sobre os benefícios e possíveis malefícios do produto nos rótulos, uma vez que a embalagem é um fator decisivo na escolha do produto.

\section{Referências}

AGÊNCIA NACIONAL DE VIGILÂNCIA SANITÁRIA - ANVISA. Resolução nº 19, de 30 de abril de 1999. Aprova o Regulamento Técnico de Procedimentos Para Registro de Alimento com Alegação de Propriedades Funcionais e ou de Saúde em sua Rotulagem. Diário Oficial da União, Brasília, 03 mai. 1999. Disponível em :http://portal.anvisa.gov.br/documents/10181/2718376/RES_18_1999_COMP.pdf/dd30fd 35-e7ea-4f8d-be72-ae2e439191b. Acessado em: 28 de julho de 2020.

AGÊNCIA NACIONAL DE VIGILÂNCIA SANITÁRIA - ANVISA. Resolução n ${ }^{\circ} 241$ de 26 de julho de 2018. Dispõe sobre os requisitos para comprovação da segurança e dos benefícios à saúde dos probióticos para uso em alimentos. Diário Oficial da União, Brasília, 27 de julho de 2018. Disponível em: http://www.in.gov.br/materia/lasset_publisher/Kujrw0TZC2Mb/content/id/34379910/do1-2018-07-27-resolucao-dadiretoria-colegiada-rdc-n-241-de-26-de-julho-de-2018-34379900 . Acessado em: 28 de julho de 2020.

BAYER, K. H. Levantamento do nível de conhecimento dos consumidores sobre os alimentos funcionais no município de Ponta Grossa (PR). Orientador: Ms. Eliana Queiroz Bortolozo, 2012, 60f. Trabalho de Conclusão de Curso (Tecnologia em Alimentos) Universidade Tecnológica Federal do Paraná, Ponta Grossa, 2012. 
BIBLIOTECA VIRTUAL EM SAÚDE. Alimentos funcionais. Disponivel em: https://bvsms.saude.gov.br/dicas-em-saude/420-alimento-funcionais. Acessado em: 27 de julho de 2020.

CRUZ, G. F. R. O comportamento do consumidor de alimentos funcionais. Orientadora: Dra. Marlette Cassia Oliveira Ferreira. 2016. 88f. Trabalho de Conclusão de Curso (Tecnologia em Processos Gerenciais) - Instituto Federal de São Paulo, Caraguatatuba, 2016.

FARIA, C. P. et al. Parâmetros de produção de leite de búfala fermentado por Lactobacillus casei. Pesquisa Agropecuária Brasileira, Brasília, v. 41, n. 3, p. 511-516, 2006.

FERREIRA, C. L. L. F.; SILVA, A. C. Probióticos e Prebióticos na Saúde da Criança. In: ROSA, C. O. B; COSTA, N. M. B. Alimentos Funcionais- Componentes Bioativos e Efeitos Fisiológicos. Rio de Janeiro: Editora Rubio, 2010, P.97-108.

GALLINA, D. A. et al. Caracterização de bebida obtida a partir de leite fermentado simbiótico adicionado de polpa de goiaba e avaliação de viabilidade das bifidobactérias. Revista do Instituto de Laticínios Cândido Tostes, Juiz de Fora, v. 67, n. 386, p. 45-54, 2012.

GALLOTII, M. E. R. Motivação e valores associados ao consumo de alimentos funcionais e nutricosméticos: um estudo exploratorio com mulheres no Rio de Janeiro. Orientadora: Letícia Moreira Casotti. 2012, 316f. Dissertação de mestrado (Administração)- Instituto COPPEAD de Administração, UFRJ, Rio de Janeiro, 2012.

GOLDIN, B. R.; GORBACH, S. L. Clinical Indications for probiotics: an overview. Clinical Infectious Diseases, Chicago, v. 46, n.1, p. 96-100, 2008.

IKEDA, A. A.; MORAES, A.; MESQUITA, G. Considerações Sobre Tendências e Oportunidades dos Alimentos Funcionais. Revista P\&D em Engenharia de Produção, Itajubá, v.8, n.2, p. 40-56, 2010.

LEITE, R.C. O Comportamento do Consumidor de Nível Superior de Produtos Lácteos Funcionais. Orientador: Dr. Jean Philippe Palma Revillion, 2011, 90f, Dissertação (Mestrado em Agronegócios) - Universidade Federal do Rio Grande do Sul, 2011.

LEUCAS, H. L. B. Efeitos benéficos de micro-organismos envolvidos na produção de leite fermentado. Orientador: Flaviano dos Santos Martins, 2012, 51f. Monografia de Especialização (Pós-Graduação em Microbiologia aplicada às Ciências ambientais e industriais) - Universidade Federal de Minas Gerais, Belo Horizonte, 2012.

LIMA, D. F. R. Influência dos hábitos alimentares e prática de atividade física no controle do peso corporal e valores sanguíneos para glicose, triglicerídeos e colesterol. Orientadora: Eliana Aparecida Fagundes Queiroz Bortolozo, 2012, 47f. Trabalho de conclusão de curso (Tecnologia em Alimentos) - Universidade Tecnológica Federal do Paraná, Ponta Grossa, 2012.

ROSA, C. O. B.; COSTA, N. M. B. (Ed). Alimentos Funcionais Componentes Bioativos e Efeitos Fisiológicos. Rio de Janeiro: Editora Rubio, 2010, 536p. 
SAAD, S. M. I. Probióticos e prebióticos: o estado da arte. Revista Brasileira de Ciências Farmacêuticas, São Paulo, v. 42, n. 1, p. 1-16, 2006.

SHAIKH, A. Demanda por probióticos cresce significativamente. Disponível em: < https://www.milkpoint.com.br/artigos/espaco-aberto/demanda-por-probioticos-crescesignificativamente-207775 />. Acesso em 22 de julho de 2020.

SCHMIDT, M. I; DUNCAN, B. B; AZEVEDO E SILVA, G; MENEZES, A. M, MONTEIRO, C. A; BARRETO, S. M, et al. Chronic noncommunicable diseases in Brazil: burden and current challenges. Lancet. 2011; 377(9781):1949-61. DOI:10.1016/S01406736(11)60135-9.

STRINGHETA, et al. Políticas de saúde e alegações de propriedades funcionais e de saúde para alimentos no Brasil. Revista Brasileira de Ciências Farmacêuticas, São Paulo, v.43, n.2, p. 181-194, 2007. DOI: 10.1590/S1516-93322007000200004. 